\title{
PHARMACOLOGICAL ROLE OF ANIONS (SULPHATE, NITRATE, OXALATE AND ACETATE) ON THE ANTIBACTERIAL ACTIVITY OF COBALT(II), COPPER(II) AND NICKEL(II) COMPLEXES WITH NICOTINOYLHYDRAZINE- DERIVED ONO, NNO AND SNO LIGANDS
}

\author{
Zahid Hussain Chohan* and Abdur Rauf \\ Department of Chemistry, Islamia University, Bahawalpur, and \\ Bahauddin Zakariya University, Multan, Pakistan
}

\begin{abstract}
Mixed ligands biologically active complexes of cobalt(II), copper(II) and nickel(II) with nicotinoylhydrazine-derived ONO, NNO and SNO donor'Schiff-base ligands having the same metal ion but different anions such as sulphate, nitrate, oxalate and acetate have been synthesised and characterised on the basis of their physical, analytical and spectral data. In order to evaluate the role of anions on their bioability, these ligands and their synthesised metal complexes with yarious anions have been screened against bacterial species such as Escherichia coli, Pseudomonas aeruginosa and Staphylococcus aureus and the title studies have proved a definative role of anions in increasing the biological activity
\end{abstract}

\section{INTRODUCTION}

The relationship1-5 between metal ions and biological systems is currently obvious and hence, is a subject of great interest for researchers. A bulk of literature6-13 has drawn attention to this fact of the most significant nature and use of metal ions for life and their role in different biochemical processes. For example, functioning of many enzymes is metal ion dependent 14,15 . The metal ion may induce by coordination a specific "Lock geometry" of the apoprotein metal binding site so that only certain substances are able to become attached to the framework formed by the result of such interaction.

The increasing interest in this area, also attracted us to investigate and enlighten the biological role16-23 of metal ions in chelation and subsequently increasing the pharmacological properties. Paralleling the same concepts, we have furthermore investigated for the first time the possible role24-26 of anions (counter part of metal ions) in biological systems and in extension to the same, now, wish to report this role of anions (sulphate, nitrate, oxalate and acetate) on biologically active metal(II) complexes of nicotinoylhydrazine-derived Schiff-base ligands reported 27 earlier by us. The present studies principally consist of the formation of various metal(II) complexes having the same metal ion but different anions represented by a general formula [M(L) $\left.{ }_{2} \mathrm{X}_{2}\right]$ where $\mathrm{M}=\mathrm{Co}(\mathrm{II}), \mathrm{Cu}(\mathrm{II})$ and $\mathrm{Ni}(\mathrm{II}), \mathrm{L}=\mathrm{L}_{1}, \mathrm{~L}_{2}$ and $\mathrm{L}_{3}$ (Fig 1) and $\mathrm{X}=\mathrm{SO}_{4}, \mathrm{NO}_{3}, \mathrm{C}_{2} \mathrm{O}_{4}$ and $\mathrm{CH}_{3} \mathrm{CO}_{2}$, and then investigating the role of anions on the antibacterial activity against bacterial species Escherichia coli, Pseudomonas aeruginosa and Staphylococcus aureus

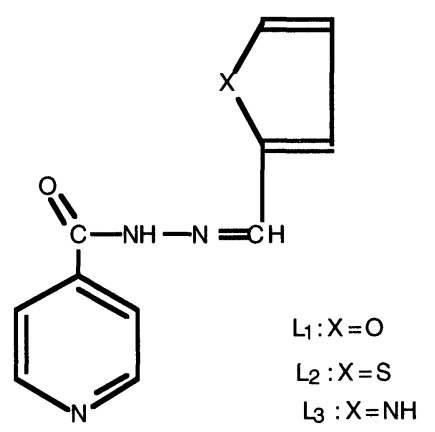

Figure 1 Structure of the ligand

\footnotetext{
* Present address for correspondence: Department of Chemistry, Meston Walk, University of Aberdeen, Old Aberdeen AB9 2UE, Scotland (U.K.)
} 


\section{EXPERIMENTAL}

Material and Methods

All solvents and chemicals used were of AR grade. All metals were used as their sulphate, nitrate, oxalate and acetate salts in the preparation of complexes. Conductance and magnetic measurements were made using a YSI model-32 conductivity bridge and Gouy balance, respectively.

Infrared spectra (nujol) were recorded in a A-10 Hitachi spectrophotometer. ${ }^{1} \mathrm{H}$ NMR spectra in DMSO $\mathrm{d}_{6}$ were obtained on a R10-Perkin Elmer spectrometer. Electronic spectra in DMF were studied on a Hitachi double-beam U-2000 model spectrophotometer using glass cells of $1 \mathrm{~cm}$ thickness. Elemental analysis of $\mathrm{C}, \mathrm{H}$, N were determined on a Coleman automatic analyser. All melting points were taken on a Gallenkamp melting point apparatus and are uncorrected. Antibacterial studies were carried out with the help of the Microbiology Laboratory, Department of Pathology, Qaid-e-Azam Medical College, Bahawalpur.

\section{Preparation of Ligands}

Nicotinoylhydrazine-derived ligands $\left(\mathrm{L}_{1}, \mathrm{~L}_{2}\right.$ and $\left.\mathrm{L}_{3}\right)$ (Figure 1) were prepared and characterised (Table 1) according to the method reported 27 earlier by us.

\section{Preparation of Metal Complexes}

To a hot $n$-butanol solution $(25 \mathrm{~mL})$ of the ligand $(0.004 \mathrm{~mol})$ was added an ethanolic solution $(15 \mathrm{~mL})$ of the metal salt $(0.002 \mathrm{~mol})$. The mixture was refluxed for $2 \mathrm{~h}$. The resulting mixture was cooled, filtered and reduced to nearly a half of its volume $(20 \mathrm{~mL})$ by an evaporator. The concentrated solution so obtained was left overnight at room temperature which resulted in the formation of a solid product. The solid product thus formed was filtered, washed with $n$-butanol $(2 \times 10 \mathrm{~mL})$ and dried. Crystallisation of the crude product in aqueous $n$-butanol (50\%) gave the desired metal complexes 1-36 (Table 2).

Antibacterial Studies

The synthesised metal complexes and ligands were screened for their antibacterial activity against bacterial species Escherichia coli (a), Pseudomonas aeruginosa (b) and Staphylococcus aureus (c). The paper disc diffusion method deviced and reported earlier17,20 by us was used for the determination of antibacterial activity.

Preparation of Discs

Metal complexes/ligands $(30 \mu \mathrm{g})$ in DMF $(0.01 \mathrm{~mL})$ were applied on a disc prepared from blotting paper ( $3 \mathrm{~mm}$ diameter) with the help of a micropipette. The discs were incubated at $37 \circ \mathrm{C}$ for $48 \mathrm{~h}$ and then applied on specific bacteria grown agar plates.

\section{Preparation of Agar Plate}

Minimal agar was used for the growth of specific bacterial species. For this purpose different media were used specifically according to the proposed method 28 of Merck.

\section{Method of Inoculation}

A platinum wire loop was used for inoculation which was first made red hot in a flame, cooled in air and then used for the application of specific bacterial strains. The preculture was prepared in $2 \mathrm{~mL}$ of nutrient broth by selecting a suitable colony of bacteria and then transfering it to the nutrient broth. It was incubated for $2 \mathrm{~h}$ at $37 \mathrm{oC}$. Then $500 \mu \mathrm{L}$ of the culture was spread on the specific agar plate and incubated for $24 \mathrm{~h}$ at $37 \mathrm{oC}^{\circ}$.

\section{Application of Discs}

A sterilised forecep was used for the application of disc on already incubated agar plates. When the disc was applied, it was then incubated at $37 \circ \mathrm{C}$ for $2 \mathrm{~h}$. The diameter of the zone of inhibition was measured.

\section{RESULTS AND DISCUSSION}

The structure of the title ligands (Table 1) was established as reported27 earlier and structure of their metal complexes synthesised in the present studies were established with the help of their physical, spectral and analytical data (Table 2). All the metal complexes were prepared by a stoichiometric reaction of metal(II) salt having different anions and the respective ligands in a molar ratio $M: L=1: 2$ (Fig 2). All complexes formed are air and moisture stable solids.

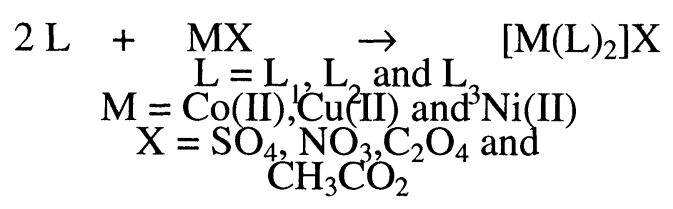


They are soluble in DMSO, DMF and water and insoluble in other solvents. The conductivity of these complexes (12-20 $\left.\mathrm{ohm}^{-1} \mathrm{~cm}^{2} \mathrm{~mol}^{-1}\right)$ in DMF shows all of them to be nonelectrolytes 28,29 . Their solubility, crystalline nature and melting behaviour suggest that they are all non-polymeric.

Table 1 Physical, Spectral and Analytical Data of Ligands

\begin{tabular}{|c|c|c|c|c|c|c|c|c|}
\hline & & Yield & M.P & IR & ${ }^{1} \mathrm{H}-\mathrm{NMR}$ & & \%)(Foun & \\
\hline & & $(\%)$ & & $\left(\mathrm{cm}^{-1}\right)$ & (ppm) & $\mathrm{C}$ & $\mathrm{H}$ & $\mathrm{N}$ \\
\hline $\mathrm{L}_{1}$ & $\mathrm{C}_{10} \mathrm{H}_{9} \mathrm{~N}_{3}$ & 46 & 157 & $\begin{array}{l}3280,2810,2575 \\
2024,1670,1620, \\
1550,1375,1112 \\
1020,970\end{array}$ & $\begin{array}{l}\text { 6.18(2H,DD,H-3,4),6.82(1H,d, } \\
\text { H-6),7.85, }(3 \mathrm{H}, \mathrm{m}, \mathrm{H}-1,4,5), 7.28 \\
\text { (2H,dd, H-2,3),8.85(1H,s, NH, } \\
\text { Exchangeable) }\end{array}$ & $\begin{array}{l}59.11 \\
(59.13)\end{array}$ & $\begin{array}{l}4.43 \\
(4.41)\end{array}$ & \\
\hline $\mathrm{L}_{2}$ & $\mathrm{C}_{10} \mathrm{H}_{9} \mathrm{~N}_{3} \mathrm{~S}$ & 50 & 149 & $\begin{array}{r}3285,2810,2570 \\
2025,1670,1620 \\
1555,1375,1115 \\
1025,970\end{array}$ & 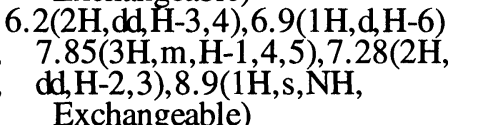 & $\begin{array}{l}59.11 \\
(59.08)\end{array}$ & $\begin{array}{l}4.43 \\
(4.48)\end{array}$ & $\begin{array}{l}20.67 \\
(20.6)\end{array}$ \\
\hline $\mathrm{L}_{3}$ & $\mathrm{C}_{10} \mathrm{H}_{10} \mathrm{~N}_{4}$ & 50 & 135 & $\begin{array}{c}3280,2815,2570 \\
2025,1670,1620 \\
1550,1375,1115 \\
1020,1010,970\end{array}$ & $\begin{array}{l}\text { 6.2(2H,dd,H-3,4),6.85(1H,d,H-6) } \\
7.9(3 \mathrm{H}, \mathrm{m}, \mathrm{H}-1,4,5), 7.25(2 \mathrm{H}, \mathrm{dd}, \\
\mathrm{H}-2,3), 8.95(2 \mathrm{H}, \mathrm{br} \mathrm{s}, \mathrm{NH} \\
\text { Exchangeable })\end{array}$ & $\begin{array}{c}64.53 \\
(64.59)\end{array}$ & $\begin{array}{l}5.37 \\
(5.38)\end{array}$ & \\
\hline
\end{tabular}

\section{Infrared Spectra}

The prominent IR frequencies of the complexes are listed in Table 2. The scrutiny of important bands exhibited by the ligands and their complexes show that a band at $3280 \mathrm{~cm}^{-1}$ in the spectra of free ligands assigned to $V(N H)$ remains unchanged in the spectra of their metal complexes thus indicating that ligands are not coordinated through -NH. However, a strong band in the spectra of ligands at $1670 \mathrm{~cm}^{-1}$ is due to $\mathrm{V}(\mathrm{C}=\mathrm{O})$ stretching distinctly shifted towards lower frequency by $30-40 \mathrm{~cm}^{-1}$ indicative of the participation of this carbonyl group in coordination. IR spectra of all ligands exhibit a band at $1620 \mathrm{~cm}^{-1}$ due to azomethine moiety which also shifted towards lower frequency by $5-10 \mathrm{~cm}^{-1}$ invariably in all the complexes suggesting respectively, that the ligands are coordinated to the metal ion via $\mathrm{C}=$ ${ }_{3} \mathrm{DN}$. In the far infrared region three bands around $375-382 \mathrm{~cm}^{-1}, 445-450 \mathrm{~cm}^{-1}$ and $510-525$ $\mathrm{cm}^{-1}$ are observed for all the complexes, which are not found in the spectrum of the free ligand. These are assigned to $\mathrm{V}(\mathrm{M}-\mathrm{S}), \mathrm{V}(\mathrm{M}-\mathrm{O})$ and $\mathrm{V}(\mathrm{M}-\mathrm{N})$ vibrations respectively, providing a conclusive evidence $30-32$ for the participation of the heteroatom of the heterocyclic ring system in coordination.

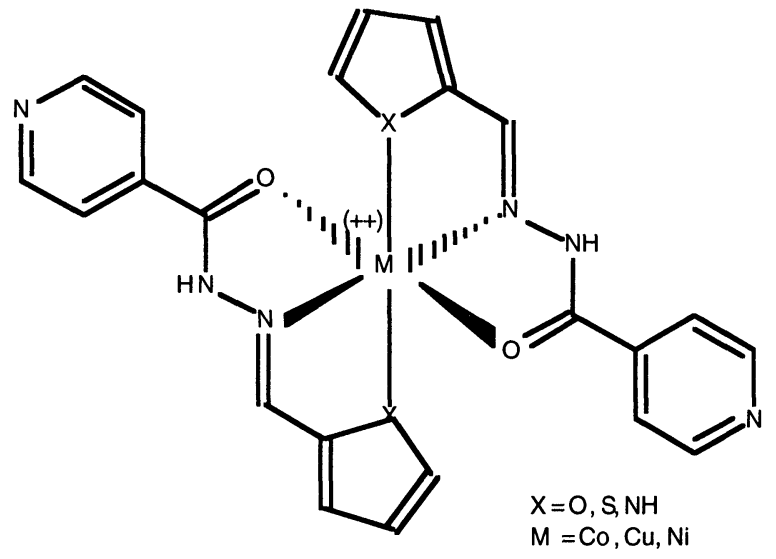

Figure 2 Proposed Structure of the Metal Complex

\section{Electronic Spectra and Magnetic Moments}

The nature of the ligand field around the metal ion and the geometry of the complexes have been deduced from the electronic spectral data and magnetic moment values given in Table 2 . $\mathrm{Co}(\mathrm{II})$ complexes show three absorption bands $4 \mathrm{~T}_{1 \mathrm{~g}}(\mathrm{~F}) \rightarrow 4 \mathrm{~T}_{2 \mathrm{~g}}(\mathrm{~F})\left(\mathrm{V}_{1}\right)$ at $8140-10215 \mathrm{~cm}^{-1}$ $4 \mathrm{~T}_{1 \mathrm{~g}}(\mathrm{~F}) \rightarrow 4 \mathrm{~A}_{2 \mathrm{~g}}(\mathrm{~F})\left(\mathrm{V}_{2}\right)$ at $17545-18770 \mathrm{~cm}^{-1}$ and $4 \mathrm{~T}_{1 \mathrm{~g}}(\mathrm{~F}) \rightarrow \mathrm{TT}_{1 \mathrm{~g}}(\mathrm{P})\left(\mathrm{V}_{3}\right)$ at $21980-23450$ $\mathrm{cm}^{-1}$ in solution having assigned 33,34 to octahedral geometry Similarly, Ni(II) complexes 
show three absorption bands between 10265-11530, 18285-19050 and 26670-29955 $\mathrm{cm}^{-1}$ corresponding to $3 \mathrm{~A}_{2 \mathrm{~g}} \rightarrow 3 \mathrm{~T}_{2 \mathrm{~g}}\left(\mathrm{~V}_{1}\right), 3 \mathrm{~A}_{2 \mathrm{~g}} \rightarrow 3 \mathrm{~T}_{1 \mathrm{~g}}(\mathrm{~F})\left(\mathrm{V}_{2}\right)$ and $3 \mathrm{~A}_{2 \mathrm{~g}} \rightarrow 3 \mathrm{~T}_{1 \mathrm{~g}}\left(\mathrm{~V}_{3}\right)$ transitions respectively, characteristic for their octahedral stereochemistry35. $\mathrm{Cu}$ (II) complexes exhibit three bands in the regions 29415-30650, 22255-23585 and 16225-18660 $\mathrm{cm}^{-1}$. The lower energy band may be assigned as the $10 \mathrm{Dq}$ band for a distorted octahedral geometry 36 corresponding to the transitions $2 \mathrm{E}_{\mathrm{g}} \rightarrow 2 \mathrm{~T}_{2 \mathrm{~g}}$. The band at $22255-23585 \mathrm{~cm}^{-1}$ may be due to intra-ligand charge transfer.

Table 2. Physical, Spectral and Analytical Data of Metal Complexes

\begin{tabular}{|c|c|c|c|c|c|c|c|c|c|}
\hline \multirow[t]{2}{*}{ No } & \multirow[t]{2}{*}{ Mol.Formula } & \multirow{2}{*}{$\begin{array}{l}\text { yield } \\
(\%)\end{array}$} & \multirow{2}{*}{$\begin{array}{l}\text { M.P. } \\
\left({ }^{\circ} \mathrm{C}\right)\end{array}$} & \multirow{2}{*}{$\begin{array}{l}\text { B.M } \\
\left(\mu_{\text {eff }}\right)\end{array}$} & \multirow{2}{*}{$\underset{\left(\mathrm{cm}^{-1}\right)}{\mathrm{IR}}$} & \multirow{2}{*}{$\begin{array}{r}\lambda_{\max } \\
\left(\mathrm{cm}^{-1}\right)\end{array}$} & \multicolumn{2}{|c|}{ Cal $(\%)$ (Found) } & \multirow[b]{2}{*}{$\mathrm{N}$} \\
\hline & & & & & & & C & $\mathrm{H}$ & \\
\hline 1 & $\begin{array}{l}{\left[\mathrm{Co}\left(\mathrm{L}_{1}\right)_{2}\left(\mathrm{SO}_{4}\right)\right]} \\
\mathrm{C}_{22} \mathrm{H}_{18} \mathrm{CoN}_{6} \mathrm{O}_{8} \mathrm{~S}\end{array}$ & 51 & $220-222$ & 4.8 & $\begin{array}{l}3280(\mathrm{NH}), 1665(\mathrm{C}=\mathrm{O}) \\
1612(\mathrm{C}=\mathrm{N}), 445(\mathrm{M}-\mathrm{O}), \\
510(\mathrm{M}-\mathrm{N}) .\end{array}$ & $\begin{array}{l}8140,18770, \\
20450 .\end{array}$ & $\begin{array}{l}45.15 \\
(45.02)\end{array}$ & $\begin{array}{l}3.07 \\
(3.39)\end{array}$ & $\begin{array}{l}14.35 \\
(14.68)\end{array}$ \\
\hline 2 & $\begin{array}{l}{\left[\mathrm{Co}\left(\mathrm{L}_{1}\right)_{2}\left(\mathrm{NO}_{3}\right)_{2}\right]} \\
\mathrm{C}_{22} \mathrm{H}_{18} \mathrm{CoN}_{8} \mathrm{O}_{10}\end{array}$ & 52 & $206-208$ & 4.91 & $\begin{array}{l}3280(\mathrm{NH}), 1652(\mathrm{C}=\mathrm{O}), \\
1615(\mathrm{C}=\mathrm{N}), 442(\mathrm{M}-\mathrm{O}), \\
525(\mathrm{M}-\mathrm{N}) .\end{array}$ & $\begin{array}{l}9115,17545, \\
21950 .\end{array}$ & $\begin{array}{l}43.09 \\
(42.88)\end{array}$ & $\begin{array}{l}2.93 \\
(3.16)\end{array}$ & $\begin{array}{l}18.26 \\
(18.54)\end{array}$ \\
\hline 3 & $\begin{array}{l}{\left[\mathrm{Co}\left(\mathrm{L}_{1}\right)_{2}\left(\mathrm{C}_{2} \mathrm{O}_{4}\right)\right]} \\
\mathrm{C}_{24} \mathrm{H}_{22} \mathrm{CoN}_{6} \mathrm{O}_{8}\end{array}$ & 50 & $217-219$ & 4.97 & $\begin{array}{l}3280(\mathrm{NH}), 1658(\mathrm{C}=\mathrm{O}), \\
1610(\mathrm{C}=\mathrm{O}), 445(\mathrm{M}-\mathrm{O}), \\
510(\mathrm{M}-\mathrm{N}) .\end{array}$ & $\begin{array}{l}8280,17670 \\
22220 .\end{array}$ & $\begin{array}{l}49.59 \\
(49.21)\end{array}$ & $\begin{array}{l}3.78 \\
(3.22)\end{array}$ & $\begin{array}{l}14.45 \\
(14.76)\end{array}$ \\
\hline 4 & $\begin{array}{l}{\left[\mathrm{Co}\left(\mathrm{L}_{1}\right)_{2}\left(\mathrm{CH}_{3} \mathrm{CO}_{2}\right)_{2}\right]} \\
\mathrm{C}_{26} \mathrm{H}_{24} \mathrm{CoN}_{6} \mathrm{O}_{8}\end{array}$ & 53 & $202-204$ & 5.22 & $\begin{array}{l}3280(\mathrm{NH}), 1643(\mathrm{C}=\mathrm{O}) \\
1610(\mathrm{C}=\mathrm{N}), 450(\mathrm{M}-\mathrm{O}) \\
510(\mathrm{M}-\mathrm{N})\end{array}$ & $\begin{array}{l}10215,18590, \\
23450 .\end{array}$ & $\begin{array}{l}51.42 \\
(51.86)\end{array}$ & $\begin{array}{l}3.95 \\
(4.22)\end{array}$ & $\begin{array}{l}13.83 \\
(13.72)\end{array}$ \\
\hline 5 & $\begin{array}{l}{\left[\mathrm{Cu}\left(\mathrm{L}_{1}\right)_{2}\left(\mathrm{SO}_{4}\right)\right]} \\
\mathrm{C}_{22} \mathrm{H}_{18} \mathrm{CuN}_{6} \mathrm{O}_{8} \mathrm{~S}\end{array}$ & 49 & $198-200$ & 1.52 & $\begin{array}{l}3280(\mathrm{NH}), 1658(\mathrm{C}=\mathrm{O}) \\
1614(\mathrm{C}=\mathrm{N}), 445(\mathrm{M}-\mathrm{O}) \\
515(\mathrm{M}-\mathrm{N})\end{array}$ & $\begin{array}{l}16250,22255, \\
30650 .\end{array}$ & $\begin{array}{l}44.79 \\
(44.38)\end{array}$ & $\begin{array}{l}3.05 \\
(2.89)\end{array}$ & $\begin{array}{l}14.24 \\
(14.33)\end{array}$ \\
\hline 6 & $\begin{array}{l}{\left[\mathrm{Cu}\left(\mathrm{L}_{1}\right)_{2}\left(\mathrm{NO}_{3}\right)_{2}\right]} \\
\mathrm{C}_{22} \mathrm{H}_{18} \mathrm{CuN}_{8} \mathrm{O}_{10}\end{array}$ & 50 & $195-197$ & 1.57 & $\begin{array}{l}3280(\mathrm{NH}), 1661(\mathrm{C}=\mathrm{O}) \\
1615(\mathrm{C}=\mathrm{N}), 445(\mathrm{M}-\mathrm{O}) \\
515(\mathrm{M}-\mathrm{N})\end{array}$ & $\begin{array}{l}17180,23585, \\
29415 .\end{array}$ & $\begin{array}{l}42.77 \\
(42.81)\end{array}$ & $\begin{array}{l}2.91 \\
(2.76)\end{array}$ & $\begin{array}{l}1813 \\
(18.01)\end{array}$ \\
\hline 7 & $\begin{array}{l}{\left[\mathrm{Cu}\left(\mathrm{L}_{1}\right)_{2}\left(\mathrm{C}_{2} \mathrm{O}_{4}\right)\right]} \\
\mathrm{C}_{24} \mathrm{H}_{22} \mathrm{CuN}_{6} \mathrm{O}_{8}\end{array}$ & 48 & $197-199$ & 1.55 & $\begin{array}{l}3280(\mathrm{NH}), 1665(\mathrm{C}=\mathrm{O}) \\
1615(\mathrm{C}=\mathrm{N}), 450(\mathrm{M}-\mathrm{O}) \\
525(\mathrm{M}-\mathrm{N})\end{array}$ & $\begin{array}{l}18215,23190, \\
30575 .\end{array}$ & $\begin{array}{l}49.20 \\
(49.67)\end{array}$ & $\begin{array}{l}3.75 \\
(3.77)\end{array}$ & $\begin{array}{l}14.33 \\
(14.28)\end{array}$ \\
\hline 8 & $\begin{array}{l}{\left[\mathrm{Cu}\left(\mathrm{L}_{1}\right)_{2}\left(\mathrm{CH}_{3} \mathrm{CO}_{2}\right)_{2}\right]} \\
\mathrm{C}_{26} \mathrm{H}_{24} \mathrm{CuN}_{6} \mathrm{O}_{8}\end{array}$ & 51 & $200-202$ & 1.58 & $\begin{array}{l}3280(\mathrm{NH}), 1640(\mathrm{C}=\mathrm{O}) \\
1612(\mathrm{C}=\mathrm{N}), 510(\mathrm{M}-\mathrm{N}) \\
445(\mathrm{M}-\mathrm{O})\end{array}$ & $\begin{array}{l}16225,23130, \\
29770 .\end{array}$ & $\begin{array}{l}51.0 \\
(51.48)\end{array}$ & $\begin{array}{l}3.92 \\
(4.51)\end{array}$ & $\begin{array}{l}13.72 \\
(13.96)\end{array}$ \\
\hline 9 & $\begin{array}{l}{\left[\mathrm{Ni}\left(\mathrm{L}_{1}\right)_{2}\left(\mathrm{SO}_{4}\right)\right]} \\
\mathrm{C}_{22} \mathrm{H}_{18} \mathrm{NiN}_{6} \mathrm{O}_{8} \mathrm{~S}\end{array}$ & 48 & $208-210$ & 3.20 & $\begin{array}{l}3280(\mathrm{NH}), 1635(\mathrm{C}=\mathrm{O}) \\
1612(\mathrm{C}=\mathrm{N}), 525(\mathrm{M}-\mathrm{N}) \\
445(\mathrm{M}-\mathrm{O})\end{array}$ & $\begin{array}{l}10265,19050, \\
26670 .\end{array}$ & $\begin{array}{l}45.16 \\
(45.55)\end{array}$ & $\begin{array}{l}3.07 \\
(2.86)\end{array}$ & $\begin{array}{l}14.35 \\
(14.18)\end{array}$ \\
\hline & $\begin{array}{l}{\left[\mathrm{Ni}\left(\mathrm{L}_{1}\right)_{2}\left(\mathrm{NO}_{3}\right)_{2}\right]} \\
\mathrm{C}_{22} \mathrm{H}_{18} \mathrm{NiN}_{8} \mathrm{O}_{10}\end{array}$ & 50 & $203-205$ & 3.46 & $\begin{array}{l}3280(\mathrm{NH}), 1642(\mathrm{C}=\mathrm{O}) \\
1610(\mathrm{C}=\mathrm{N}), 515(\mathrm{M}-\mathrm{N}) \\
450(\mathrm{M}-\mathrm{O})\end{array}$ & $\begin{array}{l}10580,18165, \\
27692 .\end{array}$ & $\begin{array}{l}43.10 \\
(43.41)\end{array}$ & $\begin{array}{l}2.93 \\
(3.11)\end{array}$ & $\begin{array}{l}18.27 \\
(18.33)\end{array}$ \\
\hline & $\begin{array}{l}{\left[\mathrm{Ni}\left(\mathrm{L}_{1}\right)_{2}\left(\mathrm{C}_{2} \mathrm{O}_{4}\right)\right.} \\
\mathrm{C}_{24} \mathrm{H}_{22} \mathrm{NiN}_{6} \mathrm{O}_{8}\end{array}$ & 48 & $199-201$ & 3.33 & $\begin{array}{l}3280(\mathrm{NH}), 1655(\mathrm{C}=\mathrm{O}) \\
1612(\mathrm{C}=\mathrm{N}), 570(\mathrm{M}-\mathrm{N}) \\
445(\mathrm{M}-\mathrm{O})\end{array}$ & $\begin{array}{l}11530,19045, \\
28115 .\end{array}$ & $\begin{array}{l}49.61 \\
(49.93)\end{array}$ & $\begin{array}{l}3.78 \\
(4.09)\end{array}$ & $\begin{array}{l}14.45 \\
(14.66)\end{array}$ \\
\hline & $\begin{array}{l}{\left[\mathrm{Ni}\left(\mathrm{L}_{1}\right)_{2}\left(\mathrm{CH}_{3} \mathrm{CO}_{2}\right)_{2}\right.} \\
\mathrm{C}_{26} \mathrm{H}_{24} \mathrm{NiN}_{6} \mathrm{O}_{8}\end{array}$ & 51 & $210-212$ & 3.50 & $\begin{array}{l}3280(\mathrm{NH}), 1675(\mathrm{C}=\mathrm{O}) \\
1615(\mathrm{C}=\mathrm{N}), 510(\mathrm{M}-\mathrm{N}) \\
450(\mathrm{M}-\mathrm{O})\end{array}$ & $\begin{array}{l}11285,18280, \\
27111 .\end{array}$ & $\begin{array}{l}51.44 \\
(51.51)\end{array}$ & $\begin{array}{l}3.95 \\
(4.21)\end{array}$ & $\begin{array}{l}13.83 \\
(14.13)\end{array}$ \\
\hline 13 & $\begin{array}{l}{\left[\mathrm{Co}\left(\mathrm{L}_{2}\right)_{2}\left(\mathrm{SO}_{4}\right)\right]} \\
\mathrm{C}_{22} \mathrm{H}_{18} \mathrm{CoN}_{6} \mathrm{O}_{6} \mathrm{~S}_{3}\end{array}$ & 52 & $201-203$ & 4.92 & $\begin{array}{l}3280(\mathrm{NH}), 1633(\mathrm{C}=\mathrm{O}) \\
1612(\mathrm{C}=\mathrm{N}), 525(\mathrm{M}-\mathrm{N}) \\
445(\mathrm{M}-\mathrm{O}), 375(\mathrm{M}-\mathrm{S})\end{array}$ & $\begin{array}{l}8575,17545 \\
22190 .\end{array}$ & $\begin{array}{l}42.80 \\
(43.26)\end{array}$ & $\begin{array}{l}2.91 \\
(3.11)\end{array}$ & $\begin{array}{l}13.60 \\
(13.28)\end{array}$ \\
\hline 14 & $\begin{array}{l}{\left[\mathrm{Co}\left(\mathrm{L}_{2}\right)_{2}\left(\mathrm{NO}_{3}\right)_{2}\right]} \\
\mathrm{C}_{22} \mathrm{H}_{18} \mathrm{CoN}_{8} \mathrm{O}_{8} \mathrm{~S}_{2}\end{array}$ & 51 & $194-196$ & 4.82 & $\begin{array}{l}3280(\mathrm{NH}), 1642(\mathrm{C}=\mathrm{O}) \\
1614(\mathrm{C}=\mathrm{N}), 515(\mathrm{M}-\mathrm{N}) \\
450(\mathrm{M}-\mathrm{O}), 375(\mathrm{M}-\mathrm{S})\end{array}$ & $\begin{array}{l}9170,18715, \\
21980 .\end{array}$ & $\begin{array}{l}40.94 \\
(41.33)\end{array}$ & $\begin{array}{l}2.78 \\
(2.59)\end{array}$ & $\begin{array}{l}17.35 \\
(17.33)\end{array}$ \\
\hline 15 & $\begin{array}{l}{\left[\mathrm{Co}\left(\mathrm{L}_{2}\right)_{2}\left(\mathrm{C}_{8} \mathrm{O}_{4}\right)\right]} \\
\mathrm{C}_{22} \mathrm{H}_{18} \mathrm{CoN}_{6} \mathrm{O}_{6} \mathrm{~S}_{2}\end{array}$ & 51 & $203-205$ & 4.97 & $\begin{array}{l}3280(\mathrm{NH}), 1652(\mathrm{C}=\mathrm{O}) \\
1610(\mathrm{C}=\mathrm{N}), 515(\mathrm{M}-\mathrm{N}) \\
442(\mathrm{M}-\mathrm{O}), 380(\mathrm{M}-\mathrm{S})\end{array}$ & $\begin{array}{l}10215,17890, \\
22262 .\end{array}$ & $\begin{array}{l}47.30 \\
(47.01)\end{array}$ & $\begin{array}{l}2.95 \\
(3.34)\end{array}$ & $\begin{array}{l}13.78 \\
(13.91)\end{array}$ \\
\hline 16 & $\begin{array}{l}{\left[\mathrm{Co}\left(\mathrm{L}_{2}\right)_{2}\left(\mathrm{CH}_{3} \mathrm{CO}_{2}\right)\right]} \\
\mathrm{C}_{26} \mathrm{H}_{24} \mathrm{CoN}_{6} \mathrm{O}_{6} \mathrm{~S}_{2}\end{array}$ & 50 & $191-193$ & 5.16 & $\begin{array}{l}3280(\mathrm{NH}), 1648(\mathrm{C}=\mathrm{O}) \\
1615(\mathrm{C}=\mathrm{N}), 525(\mathrm{M}-\mathrm{N}) \\
450(\mathrm{M}-\mathrm{O}), 380(\mathrm{M}-\mathrm{S})\end{array}$ & $\begin{array}{l}8140,17645 \\
23450 .\end{array}$ & $\begin{array}{l}48.84 \\
(42.88)\end{array}$ & $\begin{array}{l}3.75 \\
(3.93)\end{array}$ & $\begin{array}{l}13.13 \\
(12.94)\end{array}$ \\
\hline
\end{tabular}


$17\left[\mathrm{Cu}\left(\mathrm{L}_{2}\right)_{2}\left(\mathrm{SO}_{4}\right)\right]$ $\mathrm{C}_{22} \mathrm{H}_{18} \mathrm{CuN}_{6} \mathrm{O}_{6} \mathrm{~S}_{3}$

$18\left[\mathrm{Cu}\left(\mathrm{L}_{2}\right)\left(\mathrm{NO}_{3}\right)_{2}\right]$ $\mathrm{C}_{22} \mathrm{H}_{18} \mathrm{CuN}_{8} \mathrm{O}_{8} \mathrm{~S}_{2}$

$19\left[\mathrm{Cu}\left(\mathrm{L}_{2}\right)_{2}\left(\mathrm{C}_{2} \mathrm{O}_{4}\right)\right]$ $\mathrm{C}_{24} \mathrm{H}_{18} \mathrm{CuN}_{6} \mathrm{O}_{6} \mathrm{~S}_{2}$

$20\left[\mathrm{Cu}\left(\mathrm{L}_{2}\right)_{2}\left(\mathrm{CH}_{3} \mathrm{CO}_{2}\right)_{2}\right] \quad 50 \quad 208-210 \quad 1.54$ $\mathrm{C}_{26} \mathrm{H}_{24} \mathrm{CuN}_{6} \mathrm{O}_{6} \mathrm{~S}_{2}$

$21\left[\mathrm{Ni}\left(\mathrm{L}_{2}\right)_{2}\left(\mathrm{SO}_{4}\right)\right]$ $\mathrm{C}_{22} \mathrm{H}_{18} \mathrm{NiN}_{6} \mathrm{O}_{6} \mathrm{~S}_{3}$

$22\left[\mathrm{Ni}\left(\mathrm{L}_{2}\right)_{2}\left(\mathrm{NO}_{3}\right)_{2}\right]$ $\mathrm{C}_{22} \mathrm{H}_{18} \mathrm{NiN}_{8} \mathrm{O}_{8} \mathrm{~S}_{2}$

$23\left[\mathrm{Ni}\left(\mathrm{L}_{2}\right)_{2}\left(\mathrm{C}_{2} \mathrm{O}_{4}\right)\right]$ $\mathrm{C}_{24} \mathrm{H}_{18} \mathrm{NiN}_{6} \mathrm{O}_{6} \mathrm{~S}_{2}$

$24\left[\mathrm{Ni}\left(\mathrm{L}_{2}\right)_{2}\left(\mathrm{CH}_{3} \mathrm{CO}_{2}\right)_{2}\right] 48$ $\mathrm{C}_{26} \mathrm{H}_{24} \mathrm{NiN}_{6} \mathrm{O}_{6} \mathrm{~S}_{2}$

$25\left[\mathrm{Co}\left(\mathrm{L}_{3}\right)_{2}\left(\mathrm{SO}_{4}\right)\right]$ $\mathrm{C}_{22} \mathrm{H}_{20} \mathrm{CoN}_{8} \mathrm{O}_{6} \mathrm{~S}$

$26\left[\mathrm{Co}\left(\mathrm{L}_{3}\right)_{2}\left(\mathrm{NO}_{3}\right)_{2}\right]$ $\mathrm{C}_{22} \mathrm{H}_{20} \mathrm{CoN}_{10} \mathrm{O}_{8}$

$27\left[\mathrm{Co}\left(\mathrm{L}_{3}\right)_{2}\left(\mathrm{C}_{2} \mathrm{O}_{4}\right)\right]$ $\mathrm{C}_{24} \mathrm{H}_{20} \mathrm{CoN}_{8} \mathrm{O}_{6}$

$28\left[\mathrm{Co}\left(\mathrm{L}_{3}\right)_{2}\left(\mathrm{CH}_{3} \mathrm{CO}_{2}\right)\right]$ $\mathrm{C}_{26} \mathrm{H}_{26} \mathrm{CoN}_{8} \mathrm{O}_{6}$

$29\left[\mathrm{Cu}\left(\mathrm{L}_{3}\right)_{2}\left(\mathrm{SO}_{4}\right)\right]$ $\mathrm{C}_{22} \mathrm{H}_{20} \mathrm{CuN}_{8} \mathrm{O}_{6} \mathrm{~S}$

$30\left[\mathrm{Cu}\left(\mathrm{L}_{3}\right)_{2}\left(\mathrm{NO}_{3}\right)_{2}\right]$ $\mathrm{C}_{22} \mathrm{H}_{20} \mathrm{CuN}_{10} \mathrm{O}_{8}$

$31\left[\mathrm{Cu}\left(\mathrm{L}_{3}\right)_{2}\left(\mathrm{C}_{2} \mathrm{O}_{4}\right)\right]$ $\mathrm{C}_{24} \mathrm{H}_{20} \mathrm{CuN}_{8} \mathrm{O}_{6}$

$32\left[\mathrm{Cu}\left(\mathrm{L}_{3}\right)_{2}\left(\mathrm{CH}_{3} \mathrm{CO}_{2}\right)_{2}\right]$ $\mathrm{C}_{26} \mathrm{H}_{26} \mathrm{CuN}_{8} \mathrm{O}_{6}$

$33\left[\mathrm{Ni}\left(\mathrm{L}_{3}\right)_{2}\left(\mathrm{SO}_{4}\right)\right]$ $\mathrm{C}_{22} \mathrm{H}_{20} \mathrm{NiN}_{8} \mathrm{O}_{6} \mathrm{~S}$

$34\left[\mathrm{Ni}\left(\mathrm{L}_{3}\right)_{2}\left(\mathrm{NO}_{3}\right)\right]$ $\mathrm{C}_{22} \mathrm{H}_{20} \mathrm{NiN}_{10} \mathrm{O}_{8}$

$35\left[\mathrm{Ni}\left(\mathrm{L}_{3}\right)_{2}\left(\mathrm{C}_{2} \mathrm{O}_{4}\right)\right]$ $\mathrm{C}_{24} \mathrm{H}_{20} \mathrm{NiN}_{8} \mathrm{O}_{6}$

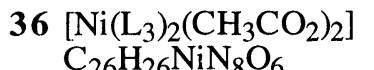

$\begin{array}{llllllll}50 & 210-212 & 1.55 & 3280(\mathrm{NH}), 1654(\mathrm{C}=\mathrm{O}), & 16780,23585,42.48 & 2.89 & 13.50\end{array}$ 1612(C=N),515(M-N), $29415 . \quad$ (42.65) (3.11) (13.47) 445(M-O),380(M-S).

$49 \quad 201-203 \quad 1.52 \quad 3280(\mathrm{NH}), 1651(\mathrm{C}=\mathrm{O}), \quad 17555,23510,40.65 \quad 2.76 \quad 17.23$ $1610(\mathrm{C}=\mathrm{N}), 525(\mathrm{M}-\mathrm{N}), \quad 30515 . \quad$ (40.77) $(3.24)(17.01)$ 445(M-O), 375(M-S).

$48 \quad 194-197 \quad 1.57 \quad 3280(\mathrm{NH}), 1635(\mathrm{C}=\mathrm{O}), \quad 16490,23135,46.95 \quad 2.93 \quad 13.68$ $1614(\mathrm{C}=\mathrm{N}), 525(\mathrm{M}-\mathrm{N}), 29715 . \quad(46.52)(3.36)(13.77)$ 450(M-O),382(M-S).

$3280(\mathrm{NH}), 1643(\mathrm{C}=\mathrm{O}), \quad 16225,22255,48.49 \quad 3.72 \quad 13.04$ $1612(\mathrm{C}=\mathrm{N}), 515(\mathrm{M}-\mathrm{N}), 29415 . \quad$ (48.67) $(3.91)(12.84)$ 450(M-O),375(M-S).

$49 \quad 199-201 \quad 3.45 \quad 3280(\mathrm{NH}), 1639(\mathrm{C}=\mathrm{O}), \quad 10265,18285,42.81 \quad 2.91 \quad 13.61$ $1610(\mathrm{C}=\mathrm{N}), 525(\mathrm{M}-\mathrm{N}), \quad 26670 . \quad(42.61)(3.38) \quad(13.77)$ 445(M-O),380(M-S).

$\begin{array}{llllllll}50 & 191-193 & 3.41 & 3280(\mathrm{NH}), 1645(\mathrm{C}=\mathrm{O}), & 11530,19050,40.96 & 2.79 & 17.36\end{array}$ $1614(\mathrm{C}=\mathrm{N}), 510(\mathrm{M}-\mathrm{N}), \quad 29955 . \quad(41.44)(2.68)(17.65)$ 450(M-O),375(M-S).

$51 \quad 201-203 \quad 3.34 \quad 3280(\mathrm{NH}), 1635(\mathrm{C}=\mathrm{O}), \quad 10880,18610,47.32 \quad 2.95 \quad 13.79$ $1612(\mathrm{C}=\mathrm{N}), 515(\mathrm{M}-\mathrm{N}), \quad 28555 . \quad(47.51)(3.26)(13.68)$ 445(M-O),382(M-S).

$\begin{array}{llllllll}48 & 210-212 & 3.48 & 3280(\mathrm{NH}), 1652(\mathrm{C}=\mathrm{O}), & 11245,19015,48.86 & 3.75 & 13.14\end{array}$ 1614(C=N),525(M-N), $26760 . \quad(48.90)(3.89) \quad(12.95)$ 450(M-O),375(M-S).

$52 \quad 190-192 \quad 4.88 \quad 3280(\mathrm{NH}), 1644(\mathrm{C}=\mathrm{O}), \quad 8140,17545, \quad 45.30 \quad 3.42 \quad 19.20$ $1612(\mathrm{C}=\mathrm{N}), 510(\mathrm{M}-\mathrm{N}), 21980 . \quad$ (45.78) (3.51) (19.06) 445(M-O).

$50 \quad 198-200 \quad 5.20 \quad 3280(\mathrm{NH}), 1661(\mathrm{C}=\mathrm{O}), \quad 10215,18770,43.23 \quad 3.27 \quad 22.90$ $1610(\mathrm{C}=\mathrm{N}), 515(\mathrm{M}-\mathrm{N}), 23450 . \quad(43.55)(3.11)(23.26)$ 450(M-O).

$51 \quad 201-203 \quad 4.92 \quad 3280(\mathrm{NH}), 1650(\mathrm{C}=0), \quad 9415,17975, \quad 50.11 \quad 3.47 \quad 19.47$ $1610(\mathrm{C}=\mathrm{N}), 515(\mathrm{M}-\mathrm{N}), \quad 22580 . \quad(50.37)(3.16) \quad(19.58)$ 450(M-O).

$52 \quad 195-197 \quad 4.86 \quad 3280(\mathrm{NH}), 1637(\mathrm{C}=\mathrm{O}), \quad 10110,18222, \quad 51.59 \quad 4.29 \quad 18.50$ $1615(\mathrm{C}=\mathrm{N}), 510(\mathrm{M}-\mathrm{N}), \quad 23145 . \quad(51.77)(4.10)(18.98)$ 445(M-O).

$49 \quad 204-206 \quad 1.57 \quad 3280(\mathrm{NH}), 1643(\mathrm{C}=\mathrm{O}), \quad 17465,22670,44.94 \quad 3.40 \quad 19.05$ $1610(\mathrm{C}=\mathrm{N}), 510(\mathrm{M}-\mathrm{N}), \quad 30550 . \quad(45.38)(3.62)(18.77)$ 445(M-O).

$50 \quad 210-212 \quad 1.55 \quad 3280(\mathrm{NH}), 1665(\mathrm{C}=0), \quad 18250,23575,42.90 \quad 3.24 \quad 22.73$ 1612(C=N),510(M-N), $29415 . \quad$ (43.21) (3.10) (22.89) $450(\mathrm{M}-\mathrm{O})$.

$48 \quad 198-201 \quad 1.52 \quad 3280(\mathrm{NH}), 1638(\mathrm{C}=\mathrm{O}), \quad 16915,22810,49.71 \quad 3.44 \quad 19.31$ $1614(\mathrm{C}=\mathrm{N}), 525(\mathrm{M}-\mathrm{N}) \quad 30650 . \quad(49.38) \quad(3.62)(19.47)$ $450(\mathrm{M}-\mathrm{O})$.

$50 \quad 207-209 \quad 1.54 \quad 3280(\mathrm{NH}), 1641(\mathrm{C}=\mathrm{O}), \quad 17580,22155,51.20 \quad 4.26 \quad 18.36$ $1612(\mathrm{C}=\mathrm{N}), 515(\mathrm{M}-\mathrm{N}), \quad 29715 . \quad(51.55)(4.11)(18.53)$ $450(\mathrm{M}-\mathrm{O})$.

$52 \quad 211-215 \quad 3.34 \quad 3280(\mathrm{NH}), 1665(\mathrm{C}=\mathrm{O}), \quad 10715,18280,45.32 \quad 3.43 \quad 19.21$ $1610(\mathrm{C}=\mathrm{N}), 515(\mathrm{M}-\mathrm{N}), \quad 27730 . \quad(45.49) \quad(2.95)(19.55)$ $450(\mathrm{M}-\mathrm{O})$.

$50 \quad 202-205 \quad 3.49 \quad 3280(\mathrm{NH}), 1648(\mathrm{C}=0), \quad 10265,19050,43.25 \quad 3.27 \quad 22.91$ 1612(C=N),525(M-N), $26670 . \quad$ (43.63) (3.14) (23.08) 445(M-O).

$51 \quad 198-200 \quad 3.38 \quad 3280(\mathrm{NH}), 1640(\mathrm{C}=\mathrm{O}), \quad 11530,18285,50.13 \quad 3.47 \quad 19.48$ $1614(\mathrm{C}=\mathrm{N}), 515(\mathrm{M}-\mathrm{N}), \quad 29955 . \quad(50.48)(3.52) \quad(19.11)$ 445(M-O).

$50 \quad 208-211 \quad 3.41 \quad 3280(\mathrm{NH}), 1663(\mathrm{C}=\mathrm{O}), \quad 10585,19045,47.44 \quad 4.66 \quad 20.11$ $1612(\mathrm{C}=\mathrm{N}), 510(\mathrm{M}-\mathrm{N}), \quad 28190 . \quad(47.61)(4.84)(19.96)$ 450(M-O).

The solid state octahedral Co(II) complexes show $\mu_{\text {eff }}$ values between 4.85-5.22 B.M, 
corresponding to a d7 electronic configuration 37 . Similarly, Ni(II) complexes show $\mu_{\text {eff }}$ values between 3.21-3.50 B.M respectively, matching fairly well with those observed for octahedral environment 38 . The magnetic susceptibility measurements for $\mathrm{Cu}$ (II) complexes show one unpaired electron per $\mathrm{Cu}$ (II) ion having $\mu_{\text {eff }}$ values $1.52-1.58 \mathrm{~B} . \mathrm{M}$ showing distorted octahedral configuration 39,40 for $\mathrm{Cu}(\mathrm{II})$ complexes.

\section{Antibacterial Properties}

Metal complexes of Schiff-base ligands lend themselves ideally to the studies of the effect of metal ions or anions: Our previous studies 16-27 in this regard give a detailed and systematic description enlightening this effect of metal ions (cations) on the pharmacological behaviour of biologically active compounds/antibacterial agent. However, in the present studies, elaboration of the role of anions (counter part of cations) in increasing the antibacterial activity in metal chelates is determined. Table 3 describes the results of these studies and it is interesting to note that when the same metal chelate/complex having different anions such as sulphate, nitrate, oxalate, and acetate was individually subjected to the evaluation of antibacterial activity, its degree of potency varied. For example, Co(II) complexes having nitrate anions as a counter part, were found to be more bactericidal than the Co(II) complexes having anions other than nitrate. Similarly, the same $\mathrm{Co}$ (II) complexes with oxalate anions were observed to be more antibacterial than the $\mathrm{Co}$ (II) complexes having acetate and sulphate anions. The identical results were found in $\mathrm{Ni}(\mathrm{II})$ and $\mathrm{Cu}(\mathrm{II})$ complexes.

\section{Table 3. Antibacterial Activity Data}

\begin{tabular}{|c|}
\hline $\begin{array}{l}\text { Ligands/ } \\
\text { Comnlexes }\end{array}$ \\
\hline
\end{tabular}

\begin{tabular}{|c|c|c|c|}
\hline $\begin{array}{l}1 \\
L^{\prime} \\
1 \\
2 \\
33 \\
4 \\
5 \\
6 \\
7 \\
8 \\
9 \\
10 \\
11 \\
12 \\
13 \\
14 \\
15 \\
16 \\
17 \\
18 \\
19 \\
20 \\
21 \\
22 \\
23 \\
24 \\
25 \\
26 \\
27 \\
28 \\
29 \\
30 \\
31 \\
32 \\
33 \\
34 \\
35 \\
36\end{array}$ & 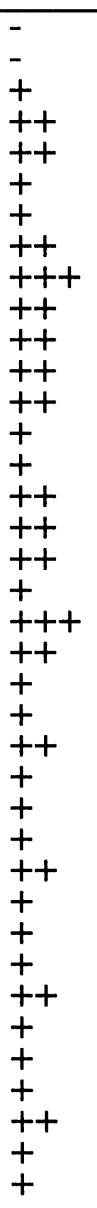 & $\begin{array}{l}+ \\
+ \\
++ \\
+++ \\
++ \\
++ \\
++ \\
+++ \\
++ \\
++ \\
++ \\
++++ \\
+++ \\
+++ \\
++ \\
+++ \\
+++ \\
+++ \\
++ \\
+++ \\
+++ \\
++ \\
++ \\
+++ \\
+++ \\
+++ \\
- \\
+++ \\
++++ \\
++ \\
+++ \\
++ \\
+++ \\
+++ \\
++ \\
++ \\
++++ \\
++++ \\
+++ \\
++\end{array}$ & $\begin{array}{l}++ \\
++ \\
+ \\
++++ \\
+++ \\
+++ \\
++ \\
+++ \\
+++ \\
+++ \\
+ \\
+++ \\
+++ \\
+++ \\
+++ \\
++++ \\
+++ \\
++ \\
++ \\
+ \\
+++ \\
+++ \\
+++ \\
+ \\
+++ \\
++ \\
+++ \\
+++ \\
+++ \\
+++ \\
+++ \\
+++ \\
++ \\
++++ \\
++ \\
+++ \\
++ \\
+++ \\
+++ \\
+++ \\
+++\end{array}$ \\
\hline
\end{tabular}

$\mathrm{a}=$ Escherichia coli, $\mathrm{b}=$ Pseudomonas aeruginosa, $\mathrm{c}=$ Staphylococcus aureus Inhibition zone diameter (mm), +: 6-10; ++: 10-14; +++: 14-18; ++++: 18-22. 
From the obtained data, it was generally observed that the order of potency in comparison to the metal complexes having chloride anions reported earlier 27 and to the present studies having anions other than chloride against the tested bacterial species was found to follow the order as

$$
\text { nitrate }>\text { oxalate }>\text { acetate }>\text { chloride }>\text { sulphate }
$$

In the light of the above observations, it is found that the role of anions in increasing the antibacterial activity of metal complexes is definite.

\section{ACKNOWLEDGEMENT}

The sincere help of Department of Pathology, Qaid-e-Azam Medical College, Bahawalpur, in undertaking the antibacterial studies is gratefully acknowledged.

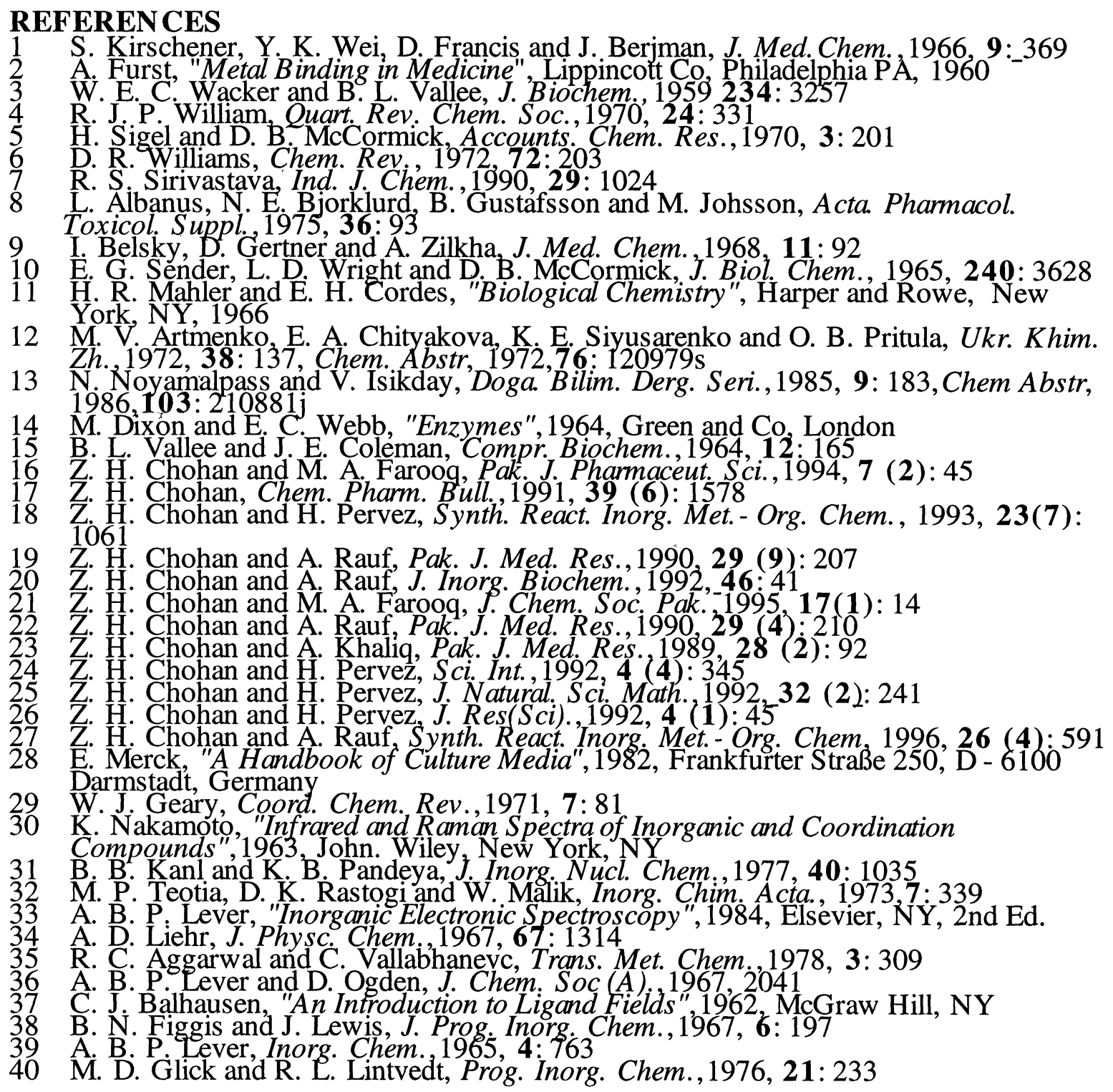

Received: September 10, 1996 - Accepted: September 17, 1996 Received in revised camera-ready format: September 23, 1996 